\title{
Hepatocellular carcinoma and hepatic cirrhosis in the west of Scotland: a 25-year necropsy review
}

\author{
R. A. BURNETT, R. S. PATRICK, W. G. S. SPILG, W. M. BUCHANAN, AND \\ R. N. M. MACSWEEN ${ }^{1}$
}

From the University of Glasgow Departments of Pathology, The Western and Royal Infirmaries, Glasgow, and from the Departments of Pathology, The Victoria, Southern General and Stobhill Hospitals, Glasgow, UK

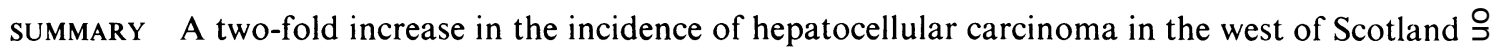
is reported on the basis of a 25 -year retrospective necropsy review (313 cases). This increase is not $\vec{T}$ accompanied by a corresponding increase in the incidence of hepatic cirrhosis. The relationship between hepatocellular carcinoma and hepatic cirrhosis is discussed in the light of these findings.

Primary hepatocellular carcinoma (PHC) is a very common tumour in some parts of the world, notably Africa and south-east Asia, but has hitherto been regarded as relatively uncommon in Europe and the Americas. However, recent reports from the west of Scotland have suggested that the incidence of this tumour is increasing (Manderson et al., 1968; MacSween, 1974) and this trend has also been reported in Europe and North America (MacDonald, 1957; Glenert, 1961 ; Patton and Horn, 1964; Ohlsson and Nordén, 1965) as well as in other parts of the United Kingdom (Elkington et al., 1963; Stone et al., 1968).

We have undertaken a retrospective review of the necropsy records of the five major Glasgow teaching general hospitals over the 25 -year period $1950-74$ and report a twofold increase in the incidence of hepatocellular carcinoma. In three of these hospitals we have compared the incidence of this tumour with the incidence of hepatic cirrhosis in the same adult necropsy population over the same 25 -year period.

\section{Results}

The necropsy records of the five Glasgow teaching hospitals (Western Infirmary, Royal Infirmary, Victoria Infirmary, Southern General Hospital, and

${ }^{1}$ Requests for reprints to: Dr R. N. M. MacSween, Department of Pathology, Western Infirmary, Glasgow G11 6NT

Received for publication 25 July 1977
Stobhill Hospital) over the 25-year period 1950-74 $\vec{\oplus}$ have included 313 cases of histologically proven 9 hepatocellular carcinoma from a total of 58074 . adult necropsies (total necropsy incidence $0.54 \%$ ). During this period the necropsy incidence of this tumour has risen steadily to twice its original level (1950-54 incidence $0.34 \% ; 1970-74$ incidence $0.73 \%)$ and this trend is similar in both sexes (male $0.27 \% \overrightarrow{\vec{B}}$ and $0.56 \%$; female $0.07 \%$ and $0.16 \%$ ) (Fig. 1). The

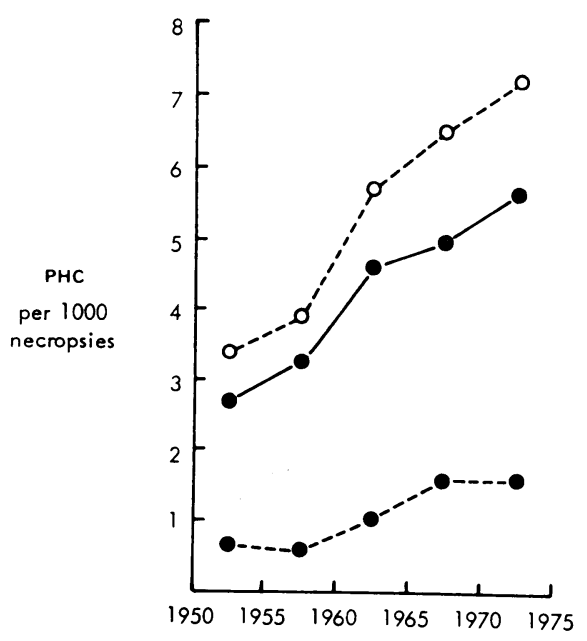

Fig. 1 Incidence of hepatocellular carcinoma $(P H C)$ over the 25-year period 1950-74. Combined necropsy figures from the five major Glasgow teaching hospitals: - - - $\mathrm{O}$, total PHC; - , male 108 
tumour was found to be almost four times more common in males (male cases $246(78.6 \%)$; female cases $67(21 \cdot 4 \%))$.

In addition, in three of these hospitals (WI, RI, and VI) we were able to compare the incidence of tumours ( 233 cases, $0.69 \%$ of total necropsies) with the incidence of hepatic cirrhosis ( 1011 cases, $2.95 \%$ of total necropsies; male 582, female 429) (Fig 2).

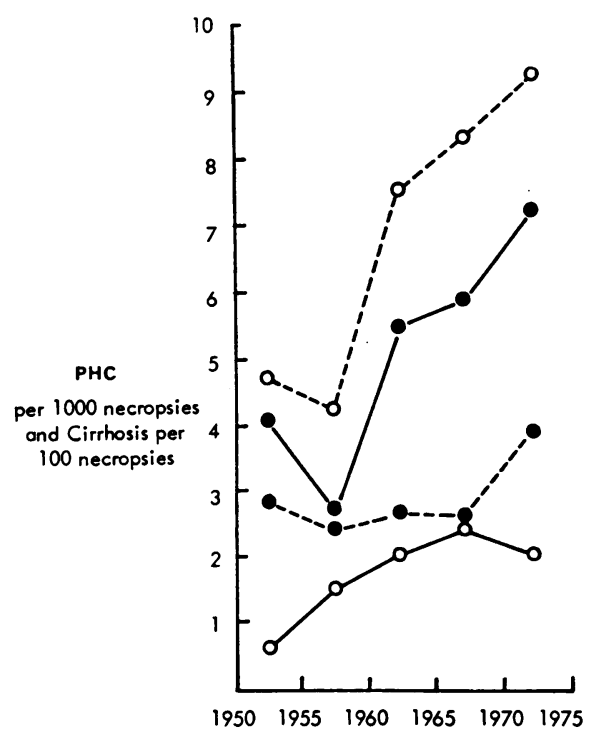

Fig. 2 Incidence of hepatic cirrhosis and hepatocellular carcinoma (PHC) over the 25-year period 1950-74. Combined necropsy figures from the Glasgow Western, Royal, and Victoria Infirmaries: $\bigcirc-\ldots--\bigcirc$, total PHC; O- male PHC; - - - - O, total cirrhosis; $\bigcirc-\bigcirc$, female PHC. Note

PHC per 1000 necropsies and cirrhosis per 100 necropsies.

The tumour/cirrhosis relationship can be examined in two ways: (a) cases of tumour associated with cirrhosis (TC) divided by total tumour cases $(\mathrm{T})$ - TC/T index, ie, the proportion of the tumour cases associated with cirrhosis; and $(b)$ cases of tumour associated with cirrhosis (TC) divided by total cirrhosis cases (C) - TC/C index, ie, the proportion of the cirrhosis cases in which tumour supervened. The overall $\mathrm{TC} / \mathrm{T}$ index in the 233 cases was $73 \%$, the corresponding male and female indices being $80 \%$ and $56 \%$ respectively. This index did not change over the period of review. The overall TC/C index was $16.8 \%$, the male and female indices being $24.4 \%$ and $6.5 \%$ respectively. This index has changed over the period of review, comparison of the first $12 \frac{1}{2}$ years with the second half indicating that it has increased by a factor of 1.51 .
A total of 61 tumours were not associated with cirrhosis (male 37, female 24) and, although the numbers are small, there has been a steady increase during the period of review $(0.06 \%$ of necropsies in $1950-54$ to $0 \cdot 2 \%$ in $1970-74$ ).

\section{Discussion}

We appreciate that there are many sources of error inherent in retrospective necropsy surveys. Changes in disease patterns in the hospital population do not necessarily give an accurate reflection of change in the general population. Furthermore the necropsy population is itself a selected group of the hospital population and there are many different factors which may influence selection over a period of as long as a quarter of a century. Nevertheless some general comments can be made. Firstly, the use of data from five major hospitals, apart from increasing the number of cases, reduces the possible effects of case referrals to specialist centres, the survey, in effect, becoming a regional one. Over the 25 -year period the necropsy rates of these five hospitals have remained relatively stable and a fairly uniform increase in the incidence of hepatocellular carcinoma has been independently demonstrated in four of these five hospitals (WI, RI, VI, and SGH). Over-diagnosis has been avoided since all the tumours included in this series have been submitted to histological reassessment and doubtful cases omitted. A cause for concern was that under-diagnosis of hepatocellular carcinoma at necropsy might be a source of error and would particularly affect tumours not associated with cirrhosis. However, this group has not increased relatively more than the tumour with cirrhosis group. In addition, one of us (RAB) examined histological sections from all livers affected by malignant tumours, primary and secondary, from one of these hospitals (SGH) over this period and failed to find a single undiagnosed case of primary hepatocellular carcinoma.

Is the increase in hepatocellular carcinoma recorded here real or not? Numerous series from Europe and North America in recent years have suggested an increase in the western world (MacDonald, 1957; Glenert, 1961; Elkington et al., 1963; Patton and Horn, 1964; Ohlsson and Nordén, 1965; Manderson et al., 1968; Stone et al., 1968; MacSween, 1974). In some of these studies it has been suggested that the increase in HPC has followed an increase in the incidence of cirrhosis, whereas in others the rise appeared to be independent of cirrhosis. In an earlier survey by one of us (MacSween, 1974), an increase in the incidence of PHC was considered to be due to a parallel increase in cirrhosis, a finding which was not found in an earlier 
west of Scotland series (Manderson et al., 1968) and which has not been confirmed in the present much larger study.

Our figures indicate that the incidence of cirrhosis has remained stable until the last half decade. In contrast, an increased incidence of PHC has occurred almost equally in both sexes and in all four subgroups, that is, males with and without cirrhosis, and females with and without cirrhosis. In consequence of the increase in the non-cirrhosis associated tumours the TC/T index has not changed.

Patton and Horn postulated that the increase in hepatocellular carcinoma in their series was due to longer survival in cirrhotic patients because of improved treatment. In the present series the mean age at death in the non-cirrhotic PHC patients was slightly greater than in the cirrhosis associated patients, 67 as compared with 63 years; and over the 25-year period no change has occurred in the mean age at death in either PHC or cirrhosis patients. These observations therefore do not support Patton and Horn's assertion, but, in the absence of comparable data on the age at clinical presentation with symptoms of cirrhosis, we cannot refute their claim. A further possibility is that the increase in PHC reflects an increase in cirrhosis, but that because of younger death in patients with PHC the carcinoma curve has risen before the cirrhosis curve. This is not supported however by our observations in that there was no difference between the mean age at death of cirrhotic and PHC patients.

That PHC is frequently associated with cirrhosis is widely accepted, but the nature of the relationship has been a matter for some speculation. Becker (1974) states 'All authors agree that the incidence of hepatoma and cirrhosis are parallel'. While it is true that the incidence of cirrhosis in any population in the western world is a good guide to the likely tumour incidence, we suggest that the true relationship between these two conditions has not been clarified because of the relative rarity of this tumour and the small numbers in most of the published series. We believe that the relationship is more complex than cirrhosis acting as a simple premalignant lesion.

It is easy to conceive that the continued hepatocellular injury of cirrhosis might predispose to tumour formation, and it has usually been assumed that the continued parenchymal damage, implicit in the cirrhosis process, was capable of provoking hepatocyte regeneration and hyperplasia to the point of autonomy. This concept is supported by the observations that PHC is more likely to develop in cirrhosis showing a macronodular pattern, often with evidence of an associated active hepatitis, these being morphological features which indicate longstanding and continuing regeneration and hyperplasia of the hepatocytes.

However, other factors obviously have to be invoked to explain, for example, the occurrence of this tumour in non-cirrhotic livers. We suggest that $\stackrel{\mathbb{\nabla}}{\circ}$ the present results are consistent with the postulate that cirrhosis tends to act as a powerful promoting agent (co-carcinogen) in the presence of a primary carcinogenic factor or factors, whether viral or $\vec{\omega}$ chemical. It is an increased exposure to this carcinogenic factor which is responsible for the increase in tumour incidence in both the cirrhotic $\dot{\omega}$ and non-cirrhotic groups. The cirrhotic state, however, enhances the effect of an as yet unidentified carcinogen, thus explaining the preponderance of $\infty$ PHC in cirrhotic livers. Furthermore, the increased $ᄋ$ exposure risk to this factor is reflected in an increas- ing malignant transformation rate (TC/C index) $\mathrm{T}$ while the overall $\mathrm{TC} / \mathrm{T}$ index has remained constant.

In conclusion it is worth observing that our hypothesis does not exclude the possibility that the same agent or agents (for example, HB virus) may, in $\vec{\theta}$ susceptible individuals, express a cirrhogenous and/ or carcinogenic effect.

\section{References}

Becker, F. F. (1974). Hepatoma-nature's model tumor. A review. American Journal of Pathology, 74, 179-200.

Elkington, S. G., McBrien, D. J., and Spencer, H. (1963). Hepatoma in cirrhosis. British Medical Journal, 2, 15011503.

Glenert, J. (1961). Primary carcinoma of the liver: a postmortem study of 104 cases. Acta Pathologica et Microbiologica Scandinavica, 53, 50-60.

MacDonald, R. A. (1957). Primary carcinoma of the liver: a clinicopathologic study of 108 cases. Archives of Internal Medicine, 99, 266-279.

MacSween, R. N. M. (1974). A clinicopathological review of 100 cases of primary malignant tumours of the liver. Journal of Clinical Pathology, 27, 669-682.

Manderson, W. G., Patrick, R. S., and Peters, E. E. (1968). Incidence of primary carcinoma of the liver in the West of Scotland between 1949 and 1965. Gut, 9, 480-484.

Ohlsson, E. G. H., and Nordén, J. G. (1965). Primary ज̂ carcinoma of the liver: a study of 121 cases. Acta N Pathologica et Microbiologica Scandinavica, 64, 430440.

Patton, R. B., and Horn, R. C. Jr. (1964), Primary liver O carcinoma: autopsy study of 60 cases. Cancer, 17, 757- 0 768.

Stone, W. D., Islam, N. R. K., and Paton, A. (1968). The $\stackrel{\oplus}{+}$ natural history of cirrhosis: experience with an unselected group of patients. Quarterly Journal of 0 Medicine, 37, 119-132. 